\title{
Effect of the imatinib treatment regimen on the
} postoperative prognosis of patients with high-risk gastrointestinal stromal tumors

This article was published in the following Dove Press journal: OncoTargets and Therapy

\author{
Yan-Shu $\mathrm{Li}^{1,2}$ \\ Wei $\mathrm{Li}^{3}$ \\ Qing-Sheng Zeng' \\ Wei-Hua Fu' \\ 'Department of General Surgery, Tianjin \\ Medical University General Hospital, \\ Tianjin, People's Republic of China; \\ ${ }^{2}$ Department of General Surgery, Huabei \\ Petroleum General Hospital, Renqiu, \\ Hebei, People's Republic of China; \\ ${ }^{3}$ Department of General Surgery, Cang \\ Zhou Central Hospital, Cangzhou, Hebei, \\ People's Republic of China
}

Background: Surgical resection is the standard treatment for localized and potentially resectable gastrointestinal stromal tumors (GISTs), If the postoperative pathology diagnosis indicates that patients are at high risk of recurrence, they should be treated with imatinib. Even though the introduction of imatinib substantially improved the outcome of GIST patients, it is unclear whether different imatinib treatment regimens affect patients' survival. Methods: This retrospective study included 120 patients who underwent tumor resection for high-risk GISTs between January 2009 and October 2018. The patients were divided into three groups: one group of patients received postoperative imatinib adjuvant therapy regularly (regular treatment group); the second group was not treated with imatinib until they were found to have disease progression (observation group); the third group was treated with postoperative imatinib adjuvant therapy irregularly (irregularly treatment group). The progression-free survival (PFS) and overall survival (OS) were compared between the three groups, and the prognostic risk factors were analysed by the Cox regression model.

Results: The median PFS was 45 months (range: 25-59). The 3- and 5-year PFS values were $71.3 \%$ and $49.9 \%$, respectively. The PFS in the regular group was longer than in the observation group and irregular group $(P<0.001)$. The median OS was 59 months (range:47-78). The 3- and 5-year OS values were $91.6 \%$ and $84.2 \%$, respectively. There were no differences in OS among the three groups $(P=0.150)$. The extent of radical resection $(P<0.001)$ and intraoperative tumor rupture $(P=0.005)$ were independent prognostic factors influencing OS.

Conclusions: Irregular administration of imatinib was associated with a worse PFS, but it did not affect the OS of patients with high-risk GISTs. Avoiding intraoperative tumor rupture and $\mathrm{R} 0$ resection were associated with better survival.

Keywords: gastrointestinal stromal tumor, imatinib, overall survival, progression-free survival, surgery

\section{Introduction}

Gastrointestinal stromal tumors (GISTs) are the most common mesenchymal tumor of the digestive system and account for less than $1 \%$ of all gastrointestinal tumors. These tumors are generally believed to be potentially malignant and less likely to have lymph node metastasis. ${ }^{1}$ For the patients with resectable high-risk GISTs, surgical resection followed by the administration of targeted therapy is the mainstay of therapy. Several risk stratification systems have been developed to estimate the risk of tumor recurrence. In multiple models, the main predictors of recurrence
Correspondence: Wei-Hua Fu Department of General Surgery, Tianjin Medical University General Hospital, 154 Anshan Road, Heping District, Tianjin 300052, People's Republic of China Tel +860226036390l

Email tjmughgs_fwh@163.com 
established were tumor mitotic rate, size, and location. ${ }^{2}$ The revised NIH consensus criteria by Joensuu et al in 2008 demonstrated that spontaneous or surgical rupture of the tumor worsened the prognosis of GIST. Incomplete resection also adversely affected the overall survival $(\mathrm{OS})^{3}$

Most GISTs (80\%) are associated with gain-of-function mutations in the KIT gene, and approximately $10 \%$ are associated with mutations in the gene that encodes platelet-derived growth factor receptor-alpha (PDGFRA). Approximately $10 \%$ of GISTs are negative for both $K I T$ and PDGFRA mutations. ${ }^{4}$ Mutations in both receptors drive downstream intracellular signaling pathways and lead to tumorigenesis. 5

Imatinib has become the first-line treatment for locally advanced/metastatic GIST and as adjuvant or neoadjuvant therapy. By competitively inhibiting the intracellular ATPbinding domain of tyrosine protein kinases, the $c$-kit gene plays a role in inhibiting intracellular signal transduction. ${ }^{6}$ All of the patients with high-risk GISTs were asked to take imatinib regularly after surgery, but we found that many patients did not comply with taking imatinib regularly during follow-up or did not take imatinib at all. To date, most were concerned with the duration of imatinib treatment and determination of whether to take imatinib. $^{7-9}$ However, no study has examined whether irregular imatinib treatment has an effect on progressionfree survival (PFS) and OS.

To shed light on this issue, we have retrospectively investigated the clinical outcome of GIST patients who took imatinib irregularly and compared the outcomes of these patients with those of GIST patients who received regular imatinib therapy and those who were not treated with imatinib.

\section{Patients and methods}

Between January 2009 and October 2018, we retrospectively gathered the clinical data from 120 consecutive patients diagnosed with GIST and treated at our institution. All patients had a histological diagnosis of GIST. The protocol of this study was approved by Tianjin Medical University General Hospital's Medical Ethics Committee and was conducted in accordance with the Declaration of Helsinki. Written informed consent was obtained from each patient.

The inclusion criteria were as follows: (1) patients with primary GISTs who were not treated with preoperative chemotherapy or imatinib, (2) postoperative tumor risk categorization considered to be high-risk GIST according to the modified National Institutes of Health (NIH) consensus classification system, (3) patients with complete information after resection and follow-up results and (4) patients without distant metastasis. The exclusion criteria were (1) patients with any other malignancy and (2) any other serious concomitant diseases that might affect survival.

In the regular group, the patients were given imatinib for at least 3 years. Once the patients suffered a recurrence or metastasis, the dose of imatinib was increased by $200 \mathrm{mg} /$ day or changed to sunitinib as second-line treatment. In the irregular group, the patients interrupted their imatinib treatment at irregular intervals; for example, the patients discontinued imatinib treatment or resumed treatment after 1 month. If the patients suffered tumor recurrence or metastasis, they received imatinib regularly. If the tumor developed further, the dose of imatinib was increased by $200 \mathrm{mg} /$ day or changed to sunitinib as second-line treatment. In the observation group, during the follow-up period, if the patients were diagnosed with metastases or relapses, they received imatinib regularly. If the disease progressed, the dose was increased by $200 \mathrm{mg} /$ day or changed to sunitinib. All patients received an initial dose of $400 \mathrm{mg} /$ day. If a mutation of KIT exon 9 was present, the dose of imatinib was increased to $600 \mathrm{mg} /$ day. In the event of intolerable complications, the dose was reduced to $300 \mathrm{mg} /$ day.

In the regular treatment group, patients who interrupted their imatinib treatment mainly because of transient toxicities or reversible concomitant illnesses but restarted their treatment within 1 month without tumor reassessment were not considered to have irregular imatinib treatment; they were kept in the regular treatment group.

\section{Follow-up}

Specially trained researchers used outpatient records, visits, letters, and telephone calls to follow-up the patients postoperatively, once every 3 months for the first 2 years, once every 6 months in the period between 3 and 5 years after surgery, and once every year thereafter. All surviving patients were followed-up for more than 5 years. The survival time was defined as the time interval from surgery to the end of the follow-up period, the time of death, or the value entered in the follow-up database (such as death from other diseases).

\section{Statistical analyses}

All statistical analyses were performed using SPSS version 22.0 (IBM Corporation, Armonk, NY, USA). Continuous 
variables were presented as median (IQR) and compared with a independent $t$-test. The categorical data were expressed as $\mathrm{N}(\%)$ and analysed by the chi-squared test. The survival rates were calculated using the Kaplan-Meier method, which used the log-rank test to detect differences in the survival curves of the various sub-groups. The Cox proportional hazards model was used to analyse the prognostic factors of multiple factors. $P$-values $<0.05$ were considered to indicate statistical significance.

\section{Results}

\section{Patients' characteristics}

The clinical and histopathological tumor characteristics of the 120 patients are presented in Table 1 . There were no differences between the three treatment groups. Among the 120 patients with high-risk GIST, there were 71 males and 49 females; their median age was 63 years (range: 23-84). The locations of the tumors were in the stomach in 44 cases $(36.7 \%)$, in the duodenum in 16 cases $(13.3 \%)$, in the jejunoileum in 49 cases $(40.8 \%)$, and in the omentum, colon, rectum, mesentery, or esophagus, in 11 cases $(9.5 \%)$. Overall, the median tumor size was $9 \mathrm{~cm}$ with a range of $2.5-32.0 \mathrm{~cm}$. There were 83 cases $(69.2 \%)$ with mitotic counts $\leq 5$ from 50 randomly selected highpower fields (HPF) and 37 cases (30.8\%) with mitotic counts $>5$ from 50 random HPF. There were 26 cases (21.7\%) with intraoperative tumor rupture and 94 cases (78.3\%) without intraoperative tumor rupture.

\section{Recurrence and survival outcome}

All patients completed the follow-up. The median followup interval was 64 months (range: 1-114). Fifty-eight patients $(48.3 \%)$ developed postoperative recurrence and/ or metastasis; In the observation group, 24 patients (75\%) developed recurrence and metastasis, and in the irregular treatment group, 23 patients (48.9\%) developed recurrence and metastasis. The recurrence rate in the irregular treatment group was higher than that in the observation group $(P<0.001)$. In the regular treatment group, 11 patients $(26.8 \%)$ developed recurrence and metastasis, and the recurrence rate was lower than that in the observation and irregular treatment groups $(P<0.001)$.

Among the 120 patients, the median PFS was 45 months (range: 25-59). The 3-and 5-year PFS was $71.3 \%$ and

Table I Patient characteristics categorized by the treatment regimen

\begin{tabular}{|c|c|c|c|c|}
\hline Characteristics & Observation group & Irregular treatment group & Regular treatment group & P-value \\
\hline Age, years (median, range) & $63(50-70)$ & $62(53-7 I)$ & $64(55-68)$ & 0.146 \\
\hline Gender & & & & 0.084 \\
\hline Male & 22 & 22 & 27 & \\
\hline Female & 10 & 25 & 14 & \\
\hline Tumor site & & & & 0.543 \\
\hline Stomach & 10 & 18 & 16 & \\
\hline Small intestine & 17 & 18 & 14 & \\
\hline Others & 5 & 11 & 11 & \\
\hline Extent of radical surgery & & & & 0.174 \\
\hline Ro & 25 & 38 & 38 & \\
\hline $\mathrm{RI}$ and $\mathrm{R} 2$ & 7 & 9 & 3 & \\
\hline Tumor diameter, cm & & & & \\
\hline$\leq 5$ & 4 & 4 & 2 & 0.608 \\
\hline$>5$ and $\leq 10$ & 17 & 20 & 20 & \\
\hline$>10$ & 11 & 23 & 19 & \\
\hline Mitotic count (/50HPF) & & & & 0.132 \\
\hline$\leq 5$ & 14 & 14 & 9 & \\
\hline$>5$ & 18 & 33 & 32 & \\
\hline Intraoperative tumor rupture & & & & 0.137 \\
\hline Yes & 10 & 11 & 5 & \\
\hline No & 22 & 36 & 36 & \\
\hline
\end{tabular}

Abbreviation: HPF, High Power Field. 
$49.9 \%$, respectively. In the regular treatment group, the median PFS was 50 months (range: 42-74). The 3-and 5-year PFS was $82.5 \%$ and $72.5 \%$, respectively. In the irregular treatment group, the median PFS was 46 months (range: 25-61). The 3-and 5-year PFS was $63.1 \%$ and $51.7 \%$, respectively. In the observation group, the median PFS was 27 months (range: 12-49). The 3-and 5-year PFS was $37.5 \%$ and $25.6 \%$, respectively. There was a significant difference among the groups $(P<0.0001$; Figure 1$)$. The median OS of the 120 patients was 59 months (range: $47-78$ ). The 3 -and 5 -year OS was $91.6 \%$ and $84.2 \%$, respectively. In the regular therapy group, the median OS was 58 months (range: 45-81). The 3- and 5-year OS was 95.1 and $86.1 \%$, respectively. In the irregular treatment group, the median OS was 63 months (range: 51-77). The 3- and 5 -year OS was 91.3 and $87 \%$, respectively. In the observation group, the median OS was 60 months (range: 46-79). The 3- and 5-year OS was $87.5 \%$ and $72 \%$, respectively. There was no significant difference of OS between the groups $(P=0.150$; Figure 2$)$.

\section{Prognostic factors}

The Kaplan-Meier analysis showed that the extent of radical resection $(P<0.001)$ and intraoperative tumor rupture $(P=0.005)$ were factors influencing OS (Table 2$)$. The multivariate Cox regression analysis showed that intraoperative tumor rupture (HR: 9.413; 95\%CI: 1.318-67.199; $P=0.025$ ) and the extent of radical surgery (HR: 7.831; 95\% CI: 1.687-36.171; $P=0.009)$ were independent predictive factors of OS.

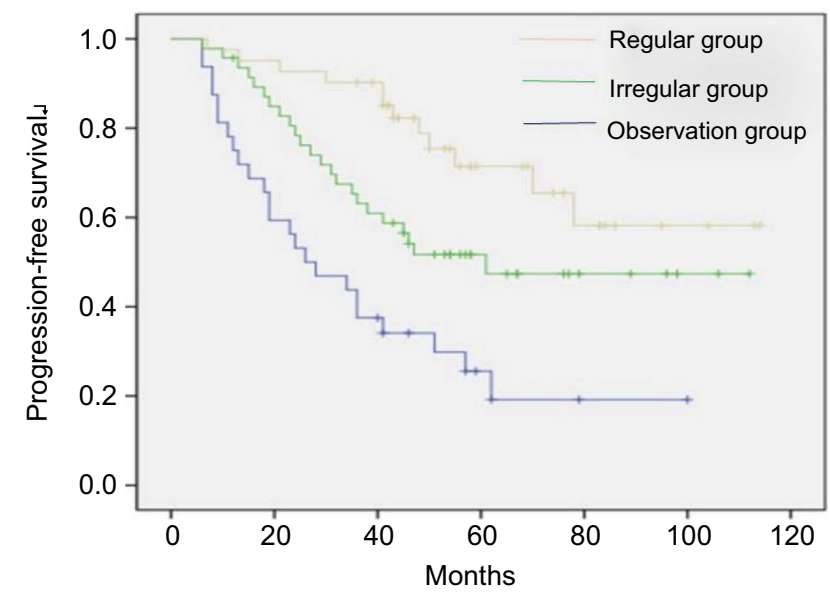

Figure I Comparison of the progression-free survival (PFS) of patients in the regular therapy group, irregular treatment group and observation group $(P<0.000 \mathrm{I})$.

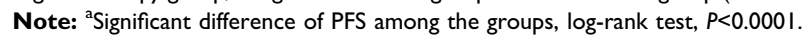

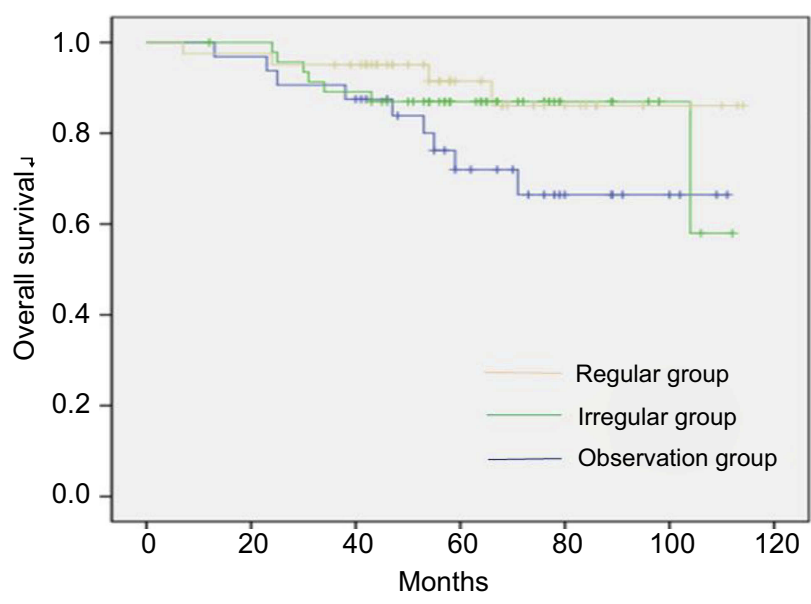

Figure 2 Comparison of the overall survival of patients in the regular therapy group, irregular treatment group and observation group $(P=0.150)$.

Notes: No significant difference of OS between the groups, log-rank test, $P=0.150$.

\section{Discussion}

GIST is a mesenchymal tumor of the digestive system with a poor response to chemotherapy and radiotherapy. However, with R0 resection, it has been documented that $80 \%$ of the patients experience tumor recurrence within 5 years after surgery and will eventually die from the disease. ${ }^{10}$ This finding is likely attributable to the persistence of microscopic disease following surgery.

Imatinib is an oral, synthetic, small-molecule tyrosine kinase inhibitor that targets the Kit and PDGFRA proteins. ${ }^{11}$ Clinical trials of gastrointestinal stromal tumors demonstrating that adjuvant imatinib therapy improves the survival benefit of patients with advanced GIST before or after surgery and in the setting of unresectable or metastatic disease. ${ }^{12-14}$ According to the Chinese consensus guidelines for the diagnosis and management of GIST, imatinib is the first-line therapy recommended for patients with intermediate or high risk gastrointestinal stromal tumor after surgical resection. The recommended adjuvant imatinib initial dosage is $400 \mathrm{mg} /$ day. ${ }^{15}$ For intermediate-risk GIST, nongastric origin GIST, such as small intestinal or colorectal GIST, shows a higher risk of recurrence compared with gastric GIST. Therefore, 3-year adjuvant imatinib therapy is recommended, whereas 1-year adjuvant imatinib therapy is recommended for intermediate-risk gastric GIST. ${ }^{16}$ Patients with high-risk GIST are advised to receive at least 3 years of adjuvant therapy. ${ }^{9,17}$

In clinical settings, a significant proportion of patients choose to temporarily discontinue therapy, either with or without supervision by their physicians, because of recurrent toxicities, economic constraints, concomitant comorbidities, 
Table 2 Univariate analysis of patients' overall survival

\begin{tabular}{|c|c|c|c|}
\hline Characteristics & $\mathbf{N}(\%)$ & Median survival (months) & $P$-value \\
\hline Gender & & & 0.388 \\
\hline Male & $71(57.1)$ & 43 & \\
\hline Female & $49(49.3)$ & 48 & \\
\hline Age (years) & & & 0.344 \\
\hline$<60$ & 44 (36.7) & 67 & \\
\hline$\geq 60$ & $76(63.3)$ & 57 & \\
\hline Tumor site & & & 0.198 \\
\hline Stomach & 44 (36.7) & 58 & \\
\hline Small intestine & $49(40.8)$ & 65 & \\
\hline Others & $27(22.5)$ & 56 & \\
\hline Extent of radical surgery & & & $<0.001$ \\
\hline Ro & I0I (84.2) & 65 & \\
\hline $\mathrm{R} 1$ and $\mathrm{R} 2$ & $19(15.8)$ & 34 & \\
\hline Tumor diameter, $\mathrm{cm}$ & & & 0.945 \\
\hline$\leq 5$ & $10(8.3)$ & 53 & \\
\hline$>5$ and $\leq 10$ & $57(47.5)$ & 59 & \\
\hline$>10$ & $53(44.2)$ & 62 & \\
\hline \multicolumn{4}{|l|}{ Mitotic count (/50HPF) } \\
\hline$\leq 5$ & $83(69.2)$ & 63 & 0.251 \\
\hline$>5$ & $37(30.8)$ & 58 & \\
\hline Intraoperative tumor rupture & & & $<0.001$ \\
\hline Yes & $26(21.7)$ & 45 & \\
\hline No & 94 (78.3) & 64 & \\
\hline
\end{tabular}

Abbreviation: HPF, High Power Field.

or desire to have a break from therapy. Thus, many studies have shed light on the effect of duration of imatinib treatment or the lack of imatinib treatment on the clinical outcome and showed that patients who received imatinib for a short duration or not at all have a lower rate of the 3- and 5-year PFS and OS..$^{718,19}$ To date, no study has examined the effect of irregular imatinib treatment on the patients' outcome. Therefore, we analysed the effects of the irregular therapy on the overall survival and PFS. The results showed that patients receiving irregular imatinib treatment have a worse PFS rate and tended to be more likely to suffer tumor recurrence or metastasis compared with the regular treatment group $(P<0.0001)$. Nevertheless, no difference of the OS rates between the groups was observed $(P=0.150)$, which may be due to their physicians treating these condition introducing or reintroducing imatinib regularly, increasing the doses of imatinib or changing to sunitinib as second-line treatment.

Adjuvant imatinib can be given according to the risk stratification. Thus, adequate risk stratification is necessary to select patients who will benefit the most from this therapy. The US National Institutes of Health (NIH) consensus system for risk classification assessment was based on tumor site, size and mitotic count. ${ }^{20}$ Many studies have shown that large tumor size and high mitotic count are associated with poor prognosis. ${ }^{14,21}$ Several current studies demonstrated that the long-term outcomes of patients with high-risk GIST improved due to prolongation of the imatinibtreatment for at least 5 years. ${ }^{22,23}$ However, an extended duration of adjuvant treatment will bring patients more economical burdens and drug sideeffect. Therefore, we need to assess what kinds of patients need prolongedduration of adjuvant imatinib treatment. In our study, extent of radical resection $(P<0.001)$ and intraoperative tumor rupture $(P=0.005)$ were independently associated with OS. Multivariate analysis showed that complete resection was an important predictive factor in the prognosis of GISTs. Based on our results, if patients did not undergo an R0 resection or there was a rupture of tumor at the time of surgery, they should extend the duration of their adjuvant imatinib treatment for more than 3 years to achieve better clinical efficacy. 
In conclusion, we demonstrated that irregular administration of imatinib was associated with a worse PFS; however, it did not influence the OS rate. Thus, whenever a tumor recurrence or metastasis is present. physicians should make every effort to treat the patients. The surgeons should minimize the likelihood of intraoperative tumor rupture and attempt to complete $\mathrm{R} 0$ resection. If a rupture of the tumor or an R0 resection could not be performed, adjuvant treatment for more than 36 months for high-risk GISTs should be administered, although the optimal treatment duration remains unclear. Complete resection, together with tumor-free resection margins and avoidance of tumor rupture and postoperative adjuvant imatinib treatment, remains the best option for a curative approach for resectable GISTs.

\section{Ethical standards}

The study was approved by Tianjin Medical University General Hospital.

\section{Disclosure}

The authors report no conflicts of interest in this work.

\section{References}

1. Majer IM, Gelderblom H, Hout WB, Gray E, Verheggen BG. Costeffectiveness of 3-year vs 1-year adjuvant therapy with imatinib in patients with high risk of gastrointestinal stromal tumour recurrence in the Netherlands; a modelling study alongside the SSGXVIII/AIO trial. $J$ Med Econ. 2013;16(9):1106-1119. doi:10.3111/13696998.2013.819357

2. Blay JY, Levard A. Adjuvant imatinib treatment in gastrointestinal stromal tumor: which risk stratification criteria and for how long? A case report. Anticancer Drugs. 2016;27(1):71-75. doi:10.1097/ CAD.0000000000000286

3. Sanchez-Hidalgo JM, Duran-Martinez M, Molero-Payan R, et al. Gastrointestinal stromal tumors: a multidisciplinary challenge. World $J$ Gastroenterol. 2018;24(18):1925-1941. doi:10.3748/wjg.v24. i18.1925

4. Nishida T, Blay JY, Hirota S, Kitagawa Y, Kang Y-K. The standard diagnosis, treatment, and follow-up of gastrointestinal stromal tumors based on guidelines. Gastric Cancer. 2016;19(1):3-14. doi:10.1007/ s10120-015-0526-8

5. Mei L, Du W, Idowu M, von Mehren M, Boikos SA. Advances and challenges on management of gastrointestinal stromal tumors. Front Oncol. 2018;8:135. doi:10.3389/fonc.2018.00135

6. Linch M, Claus J, Benson C. Update on imatinib for gastrointestinal stromal tumors: duration of treatment. Onco Targets Ther. 2013;6:1011-1023. doi:10.2147/OTT.S31260
7. Lin JX, Chen QF, Zheng CH, et al. Is 3-years duration of adjuvant imatinib mesylate treatment sufficient for patients with high-risk gastrointestinal stromal tumor? A study based on long-term follow-up. J Cancer Res Clin Oncol. 2017;143(4):727-734. doi:10.1007/s00432-016-2334-x

8. Kurtovic-Kozaric A, Kugic A, Hasic A, et al. Long-term outcome of GIST patients treated with delayed imatinib therapy. Eur J Cancer. 2017;78:118-121. doi:10.1016/j.ejca.2017.03.024

9. Li J, Gong JF, Wu AW, Shen L. Post-operative imatinib in patients with intermediate or high risk gastrointestinal stromal tumor. Eur J Surg Oncol. 2011;37(4):319-324. doi:10.1016/j.ejso.2011.01.005

10. Rutkowski P, Bylina E, Wozniak A, et al. Validation of the Joensuu risk criteria for primary resectable gastrointestinal stromal tumour the impact of tumour rupture on patient outcomes. Eur J Surg Oncol. 2011;37(10):890-896. doi:10.1016/j.ejso.2011.06.005

11. Koumarianou A, Economopoulou P, Katsaounis P, et al. Gastrointestinal stromal tumors (GIST): a prospective analysis and an update on biomarkers and current treatment concepts. Biomark Cancer. 2015;7(Suppl 1):1-7. doi:10.4137/BIC.S25045

12. Singer S, Rubin BP, Lux ML, et al. Prognostic value of KIT mutation type, mitotic activity, and histologic subtype in gastrointestinal stromal tumors. J Clin Oncol. 2002;20(18):3898-3905. doi:10.1200/JCO.2002.03.095

13. Aparicio T, Boige V, Sabourin JC, et al. Prognostic factors after surgery of primary resectable gastrointestinal stromal tumours. Eur J Surg Oncol. 2004;30(10):1098-1103. doi:10.1016/j.ejso.2004.06.016

14. Kim TW, Lee H, Kang YK, et al. Prognostic significance of $c$-kit mutation in localized gastrointestinal stromal tumors. Clin Cancer Res. 2004;10(9):3076-3081.

15. Li J, Ye Y, Wang J, et al. Chinese consensus guidelines for diagnosis and management of gastrointestinal stromal tumor. Chin J Cancer Res. 2017;29(4):281-293. doi:10.21147/j.issn.1000-9604.2017.04.01

16. von Mehren M, Randall RL, Benjamin RS, et al. Soft tissue sarcoma, version 2.2014. J Natl Compr Canc Netw. 2014;12(4):473-483.

17. Joensuu H, Eriksson M, Hall KS, et al. One vs three years of adjuvant imatinib for operable gastrointestinal stromal tumor: a randomized trial. Jama. 2012;307(12):1265-1272. doi:10.1001/jama.2012.347

18. Lei C, Zhao B, Wang Q, Ge L, Wang H. Imatinib therapy after resection of high-risk gastrointestinal stromal tumors in Chinese patients: a median follow-up of 48 months. J Buon. 2018;23(2):460-467.

19. Lee JL, Ryu MH, Chang HM, et al. Clinical outcome in gastrointestinal stromal tumor patients who interrupted imatinib after achieving stable disease or better response. Jpn J Clin Oncol. 2006;36 (11):704-711. doi:10.1093/jjco/hyl088

20. Fletcher CD, Berman JJ, Corless C, et al. Diagnosis of gastrointestinal stromal tumors: a consensus approach. Int J Surg Pathol. 2002;10(2):81-89. doi:10.1177/106689690201000201

21. Martín J, Poveda A, Llombart-Bosch A, et al. Deletions affecting codons 557-558 of the $c$-KIT gene indicate a poor prognosis in patients with completely resected gastrointestinal stromal tumors: a study by the Spanish group for sarcoma research (GEIS). J Clin Oncol. 2005;23(25):6190-6198. doi:10.1200/JCO.2005.19.554

22. Langer C, Gunawan B, Schüler P, Huber W, Füzesi L, Becker H. Prognostic factors influencing surgical management and outcome of gastrointestinal stromal tumours. Br J Surg. 2003;90(3):332-339. doi: $10.1002 /$ bjs. 4046

23. Hsu KH, Yang TM, Shan YS, Lin P-W. Tumor size is a major determinant of recurrence in patients with resectable gastrointestinal stromal tumor. Am J Surg. 2007;194(2):148-152. doi:10.1016/j. amjsurg.2006.10.033 


\section{Publish your work in this journal}

OncoTargets and Therapy is an international, peer-reviewed, open access journal focusing on the pathological basis of all cancers, potential targets for therapy and treatment protocols employed to improve the management of cancer patients. The journal also focuses on the impact of management programs and new therapeutic

Submit your manuscript here: https://www.dovepress.com/oncotargets-and-therapy-journa agents and protocols on patient perspectives such as quality of life, adherence and satisfaction. The manuscript management system is completely online and includes a very quick and fair peer-review system, which is all easy to use. Visit http://www.dovepress.com/ testimonials.php to read real quotes from published authors. 